\title{
Compact collimators for high brightness blue LEDs using dielectric multilayers
}

\author{
Hugo J. Cornelissen ${ }^{1}$, Haiyan $\mathrm{Ma}^{2}$, Chenhung $\mathrm{Ho}^{2}$, Meijie $\mathrm{Li}^{2}$, Cong $\mathrm{Mu}^{2}$ \\ ${ }^{1}$ Philips Research Europe, High Tech Campus 34, 5656AE Eindhoven, the Netherlands, \\ ${ }^{2}$ Optics Research Group, Delft University of Technology, the Netherlands
}

\begin{abstract}
A novel method is presented to inject the light of millimeter-sized high-brightness blue LEDs into light guides of submillimeter thickness. Use is made of an interference filter that is designed to pass only those modes that will propagate in the light guide by total internal reflection. Other modes are reflected back to the LED cavity and recycled, leading to an increased brightness.

With this method a collimator has been designed and made that is only $1 \mathrm{~mm}$ thick, with a diameter of $6.5 \mathrm{~mm}$. It creates a beam of 26deg Full Width at Half Maximum. Presently, collimators with these characteristics have a thickness of 10$20 \mathrm{~mm}$ and a diameter of $20-30 \mathrm{~mm}$ and require careful mounting and alignment. The new collimator contains a 4.5micron thick interference filter made of 54 layers of $\mathrm{Nb}_{2} \mathrm{O}_{5}$ and $\mathrm{SiO}_{2}$ layers. The filter is optically coupled to the LED with Silicone adhesive which makes the configuration very robust. A cylindrical lightguide, tapered from $6.5 \mathrm{~mm}$ to $2.5 \mathrm{~mm}$ diameter and $1 \mathrm{~mm}$ thick captures the light that passes the filter, folds the light path and redirects the beam. Measurements on collimator prototypes show good agreement with the designed characteristics. This promising approach enables much more compact collimators optics that offer material cost savings and design freedom.
\end{abstract}

Keywords: thin-film interference filter; dielectric multilayer; LED; collimator; Total Internal Reflection; light guide;

\section{INTRODUCTION}

High-brightness LEDs are energy efficient and compact light sources that are widely used nowadays in various lighting applications. ${ }^{[1]}$ They provide high luminous flux and high luminance in a cost effective manner. The angular beam profile of an LED is often a broad Lambertian distribution. In order to increase the illuminance at a certain target surface, additional collimator optics is often used, see Fig. 1 for some examples. Whereas the efficiency of the commercial collimator optics is high, the size is often not optimally small. This presents a problem in those applications where one needs to combine many sources in a small volume, like in a projection display or a spot light module. We studied a new optical design that could lead to much more compact but equally efficient collimation optics. In the second part of this paper we introduce a concept for a large-area, multi-LED, scalable collimator based on the same principle of angular filtering. Experimental results on a prototype show good agreement with the designed performance.

\subsection{Principle of operation}

A schematic drawing of the new optics configuration is given in Fig. 2. Starting point is a Luxeon Rebel from Philips Lumileds, which has a $1 \times 1 \mathrm{~mm}^{2}$ emitting area. The top InGaN surface is rough to enhance light extraction efficiency. The original overmolded silicone dome was removed and the flat LED surface was brought in optical contact with a light guide by means of a silicone adhesive.
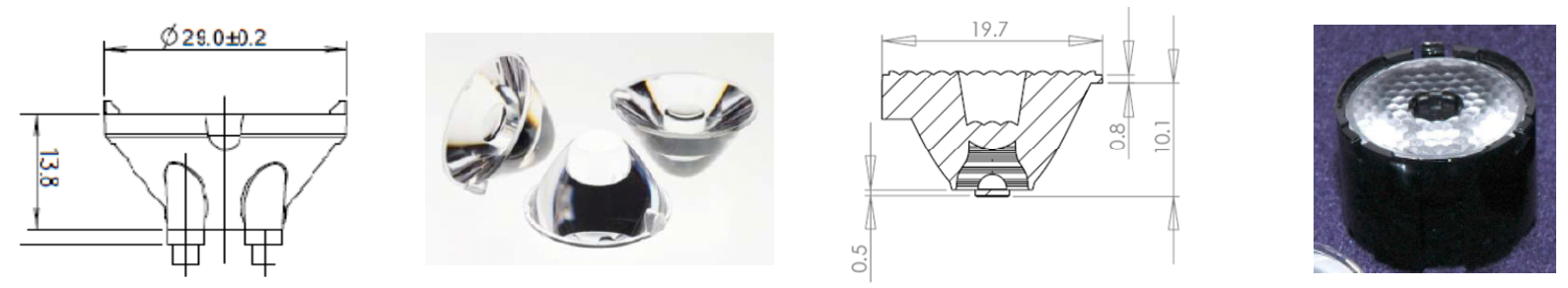

Fig. 1 Commercial collimator optics for high power LEDs (http://www.carclo-optics.co.uk, http://www.fraensrl.com/images/Fraen_FLP_Rebel_datasheet.pdf).

Eleventh International Conference on Solid State Lighting, edited by Matthew H. Kane, Christian Wetzel, Jian-Jang Huang, Proc. of SPIE Vol. 8123, 81230J - (c) 2011 SPIE · CCC code: 0277-786X/11/\$18 · doi: 10.1117/12.894977 


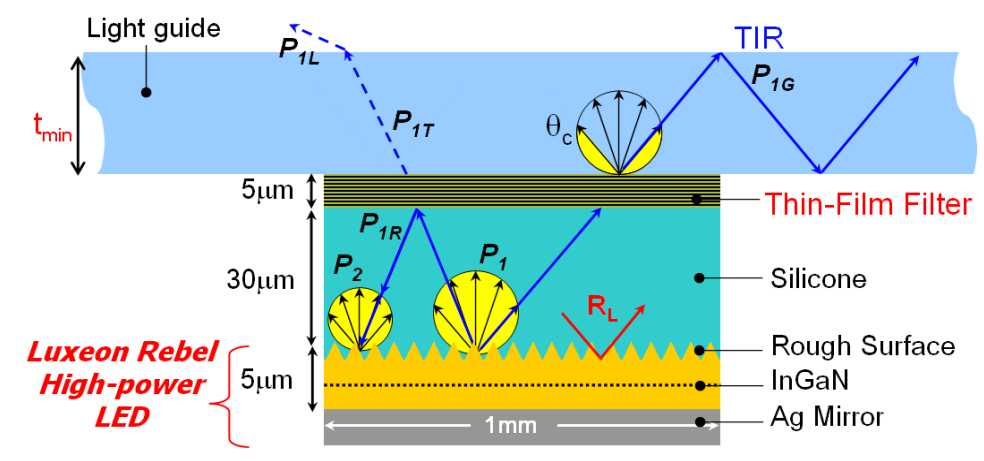

Fig. 2 The new optical configuration for coupling LEDs to thin light guides (not drawn to scale).

The most important aspect of our method is a dielectric multilayer that acts as a angular filter between the LED and the bottom of the light guide. ${ }^{[2]}$ The filter is optimized to only transmit light emitted from the LED at angles larger than the critical angle, $\theta_{\mathrm{c}}$ at the light guide-air interface. Most of the light emitted at smaller angles is reflected back towards the rough InGaN surface where it is subsequently reflected and redistributed.

In the design of the multilayer filter, the maximum number of layers was set at sixty layers and only two available materials were used, $\mathrm{Nb}_{2} \mathrm{O}_{5}$ and $\mathrm{SiO}_{2}$. Commercial software for thin film optical design, Essential MacLeod ${ }^{[3]}$ was used to optimize the performance of the filter. The targeted performance was a minimum transmission of the filter for angles below the critical angle, and a maximum transmission above this angle. In Fig. 3 the angular dependent transmission of the final 54-layer design is plotted for a wavelength of 460nm light.

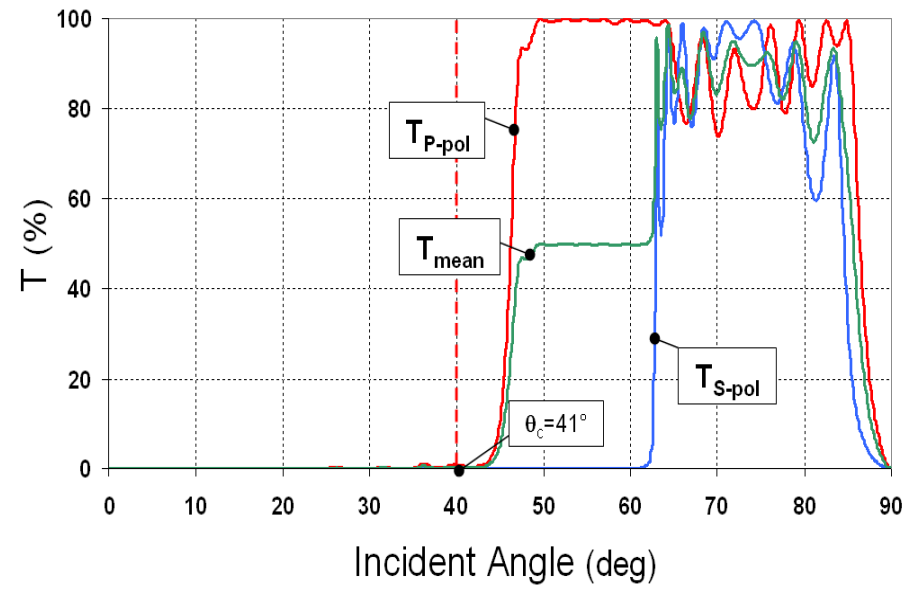

Fig. 3 Calculated optimized transmittance at $460 \mathrm{~nm}$ as a function of incidence angle.

Red curve: P-polarized; Blue curve: S-polarized; Green curve: mean.

\section{COMPACT COLLIMATOR}

A very compact collimator can be designed based on the angular filter approach. A filter as deposited on a $1 \mathrm{x} 1 \mathrm{~mm}^{2}$ $150 \mu \mathrm{m}$ thick glass substrate is mounted on an LED with silicone adhesive. A circular disk light guide made from PMMA is cone shaped with a facet at $26^{\circ}$ angle, as illustrated in Figure 4, and mounted onto the filter glass. The PMMA piece is $1 \mathrm{~mm}$ thick and tapers from $2.5 \mathrm{~mm}$ to $6.5 \mathrm{~mm}$ diameter. For these prototypes, a highly reflecting sheet was folded around the cone, or an aluminum coating was sputtered on the PMMA cone. The luminous intensity distribution of the prototypes is measured with a Source Imaging Goniometer from Radiant Imaging and favorably compares with the results from a LightTools ray tracing model as shown in Figure 5. The beam is measured to have a Full Width at Half Maximum (FWHM) of $2 \times 13^{\circ}$. Our approach provides a compact alternative to the method presented in Ref.[4] where light is recycled through the LED source using reflective micro-facets. In the present case thin-film optics is applied, exploiting the relatively narrow spectral bandwidth of the LED emission. 

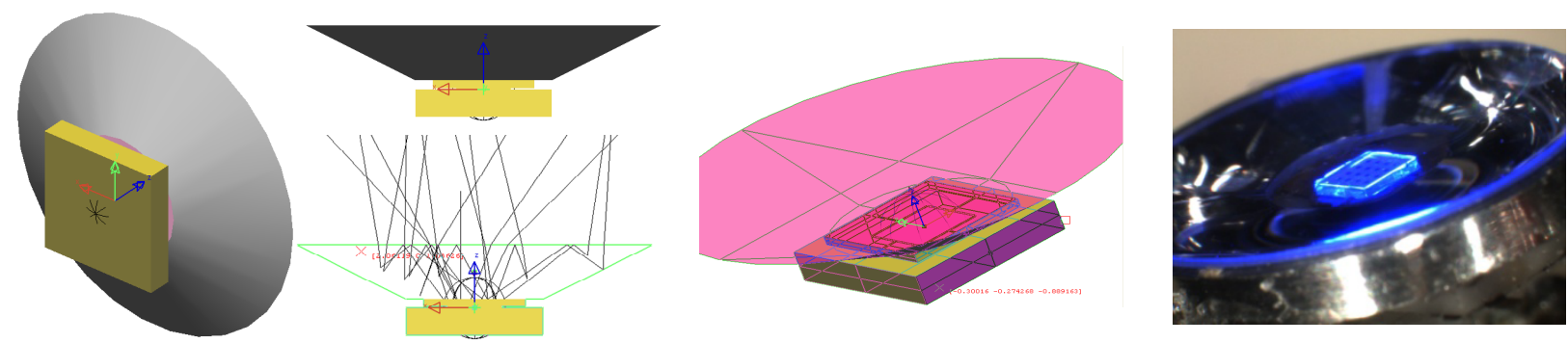

Figure 4 LightTools model of a compact collimator and a microscope picture of a prototype.
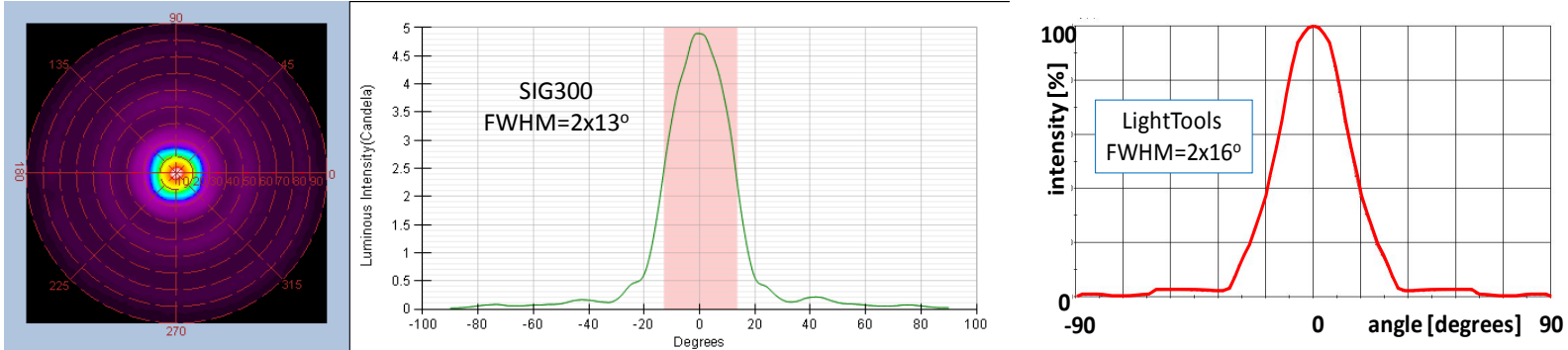

Figure 5 Optical characterization of the collimator prototype. The measured luminous intensity distribution is compared with the results from the LightTools model.

The spatial luminance distribution as measured with a luminance camera along the surface normal is shown in Figure 6, alongside with a calculated one, using backward ray tracing. It shows fair agreement with the experiment where more stray light or scattering is observed at the edges of the PMMA and filter. The light is seen to emerge from a ring shaped area, not spanning the full exit area. If the full $6.5 \mathrm{~mm}$ diameter exit area were used, the étendue-limited FWHM would be estimated as $2 \times 8.5^{\circ}$. Instead, as shown in Figure 5, the measured FWHM is $2 \times 13^{\circ}$, which suggests that the optical design of the collimator might be improved. This is explored by ray tracing modeling as illustrated in the next section, where the relatively simple single facet cone has been replaced by a more intricate curved shape.

For the present prototypes the measured efficiency was $50 \pm 5 \%$ which is caused by the relatively low reflection coefficient of the LED surface of $65 \%$. It is expected that the reflectivity of the LED surface will be improved in the future, increasing the recycling efficiency to more than $80 \%$.
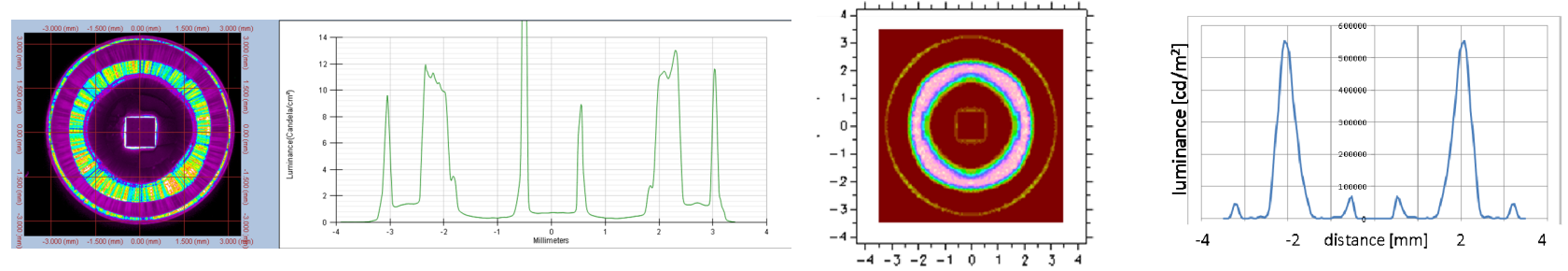

Figure 6 Spatial luminance distribution as observed along the normal with a cross-section along a horizontal line, measured (left) and calculated with backward ray tracing (right).

\section{IMPROVED COLLIMATOR OPTICS}

In the simulations, the relatively simple cone shape has been replaced by a more complex design as shown in Fig. 7 and Fig. 8. By changing to a double parabolic reflector or to a multi-facetted reflector the beam profile can be tuned to achieve a more favorable distribution. The angular distribution of the LED emission is now separated in three parts. Rays emitted at angles close to the normal and below the critical angle are reflected by the dielectric multilayer filter and recycled in the LED cavity. Rays with direction larger than the critical angle but smaller than $70^{\circ}$ pass the filter but they 


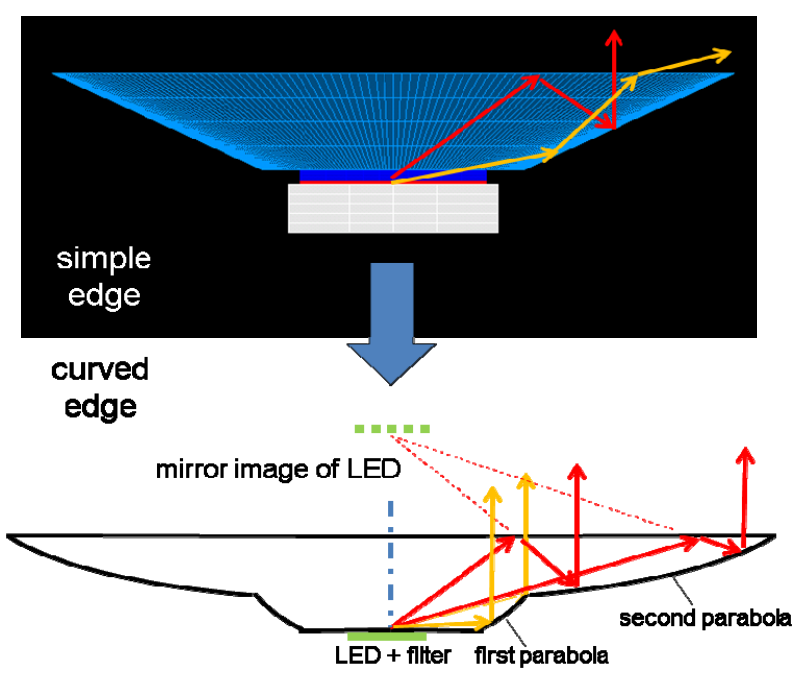

Fig. 7 Optical design for an improved beam profile.
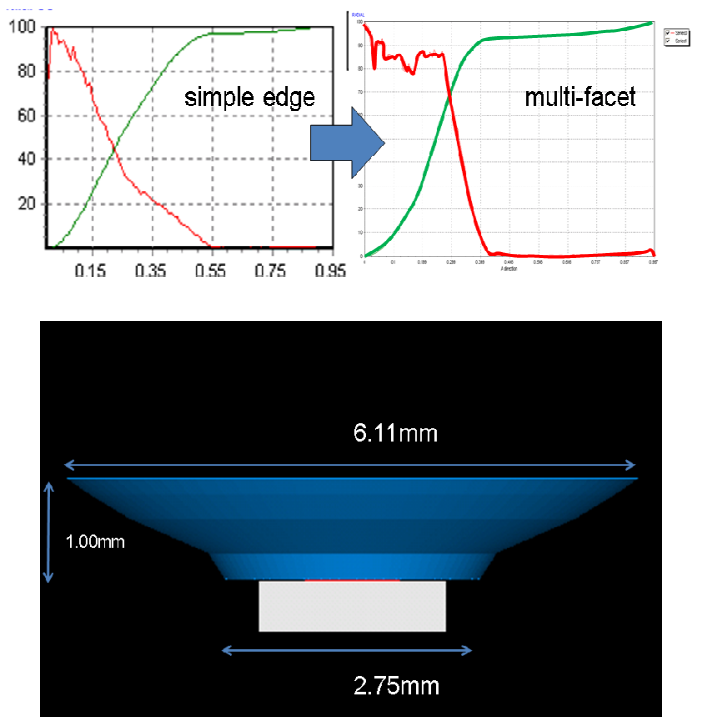

Fig. 8 Multi-facet collimator and calculated beam profile

are reflected by Total Internal Reflection at the top surface of the light guide as indicated with the red arrows in Fig. 7. They are collimated by the second parabola. Rays with emission angles larger than $70^{\circ}$ are reflected by the first parabola.

In Fig. 8 the result is shown of a multi-facet reflector design with the calculated beam profile. The red curves show a comparison of the beam profiles of the simple edge collimator and the multi-facet collimator. The former has a more peaked profile with some tailing at large angles, whereas the latter has a more flat beam profile. The green curves show the encircle energy of the beam. In the new design, more energy will be inside the FWHM of the beam.

\section{IMPROVED ANGULAR FILTER DESIGN}

As described above, an LED-masking filter consisting of 54 layers of $\mathrm{Nb}_{2} \mathrm{O}_{5}\left(\mathrm{n}_{\mathrm{H}}=2.46\right)$ and $\mathrm{SiO}_{2}\left(\mathrm{n}_{\mathrm{L}}=1.46\right)$ was designed and optimized using the thin film optical design software Essential Macleod. As shown in Fig. 3, the transmittance performance at the peak wavelength of $460 \mathrm{~nm}$ exhibits a large difference between s- and p- polarized light, whereby the output coupling efficiency is reduced. This polarization dependency stems from the Brewster angle effect at oblique incidence, depending on the unequal optical admittances for s- and p- polarized light. ${ }^{[3]}$ The transmittance difference for both polarization states is proportional to the refractive index ratio of the materials. To improve the polarization equality two possible solutions have been explored. One is to use materials with lower index ratio for which we propose polymeric instead of the inorganic materials bi-layers. The other one is to add a third material with refractive index inbetween the previous two, which results in lower index ratios.

\subsection{Design of a Polymeric Multilayer Angular Filter}

A polymeric multilayer was designed using multiple bi-layer stacks, whereby each stack consists of $m$ times of repeated unit cell $(\mathrm{HL})^{\mathrm{m}}$, as shown in Figure 9. H and L represent quarter-wave layers of the high and low index materials respectively. According to Refs. [5] and [6], for the polymeric material preferably PMMA $\left(\mathrm{n}_{\mathrm{L}}=1.49\right)$ and PEN $\left(\mathrm{n}_{\mathrm{H}}=1.75\right)$ are used to achieve a lower index ratio of 1.17, compared with the previous one of 1.68. The HL layer for each stack does not have to be exactly a quarter wavelength thick. Essentially, by proper thickness tuning, each stack can reflect light of a different part of the spectrum. Consequently, the combination of bi-layer stacks with consecutive reflection bands is capable to broaden the overall stop bandwidth, as seen in Figure 10. This broader band filter can effectively cut off (reflect) the blue LED light with small incident angle. As the transmittance spectrum will shift to shorter wavelength with increasing incident angles, when arriving at the critical angle, the filter begins to transmit LED light and keeps doing so until $90^{\circ}$.

An isotropic polymeric multilayer LED-masking angular filter made of PEN and PMMA was designed in Essential Macleod. The filter was designed with seven bi-layer stacks and has a total thickness of $55.1 \mu \mathrm{m}$. The transmittance 


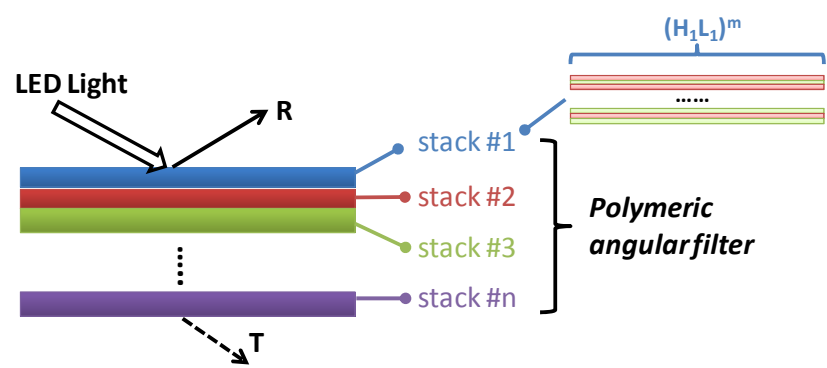

Figure 9 The structure illustration for the polymeric multilayer angular filter made of bi-layer stacks

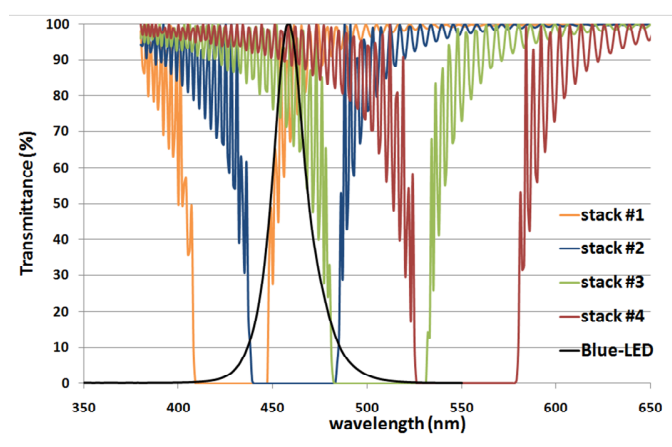

Figure 10 Transmission spectra of 4 bi-layer stacks. The black curve shows the LED spectrum.

regarding to incident angle at the central peak at 460nm is shown in Figure 11 for both polarization states. The s- and pangular edge difference is minimized to $7^{\circ}$ compared with the previous $17^{\circ}$, which enhances the efficiency as shown in section 4.3.

A complete elimination of the polarization dependency was achieved by using a birefringent polymeric multilayer to arbitrary control the Brewster angle as described in Ref. [7], where the reflectance of p-polarized light increases identically with s-polarized light. This birefringent polymeric LED-masking filter was designed with the combination of two birefringent polymeric materials with a $\mathrm{z}$-index mismatch. One possible combination could be $\mathrm{n}_{1 \mathrm{x}}=1.65, \mathrm{n}_{1 \mathrm{y}}=1.50$, $\mathrm{n}_{1 \mathrm{z}}=1.75 ; \mathrm{n}_{2 \mathrm{x}}=1.75, \mathrm{n}_{2 \mathrm{y}}=1.75, \mathrm{n}_{2 \mathrm{z}}=1.50$. As seen from Figure 12, the transmittance curves at 460nm show an angular edge difference of less than $1^{\circ}$. This filter not only perfectly achieves polarization equality at large angles, but also blocks the light at small incident angles. The decrease of the mean transmittance from $80^{\circ}$ to $90^{\circ}$ results from the large number of polymeric layers in the combined stacks, in total approximately 700. Such a birefringent polymeric angular filter can possibly be manufactured with the technology that is described in Ref. [7].

\subsection{Design of a three-material filter (HML)}

The high refractive index ratio of $\mathrm{Nb}_{2} \mathrm{O}_{5}\left(\mathrm{n}_{\mathrm{H}}=2.46\right)$ and $\mathrm{SiO}_{2}\left(\mathrm{n}_{\mathrm{L}}=1.46\right)$ leads to the wide stop band but also to the transmittance gap between both polarization states. Using a third intermediate index material (M) allows for lower index ratio of $\mathrm{n}_{\mathrm{H}} / \mathrm{n}_{\mathrm{M}}$ and $\mathrm{n}_{\mathrm{M}} / \mathrm{n}_{\mathrm{L}}$, and additionally can help to suppress the higher order harmonics of p-polarized light at large incident angle. The added third material also provides more degrees of freedom for filter optimization. Practically, we kept using the previous two high and low materials and selected an intermediate material $\mathrm{Y}_{2} \mathrm{O}_{3}$, with refractive index of $\mathrm{n}_{\mathrm{M}}=1.80$ at $460 \mathrm{~nm}$. In Essential Macleod, the transmittance of s- and p- polarized light for various wavelength and incident angles was optimized. Weights and targets were set individually corresponding to the LED spectrum with inhomogeneous incident angle ranges. A fully automatic optimization process does not lead to satisfactory results. Instead, modification of the target settings and weights are required during the optimization process. The final filter performance of the HML LED-masking filter is illustrated in Figure 13, with a total physical thickness of 6.94um. The

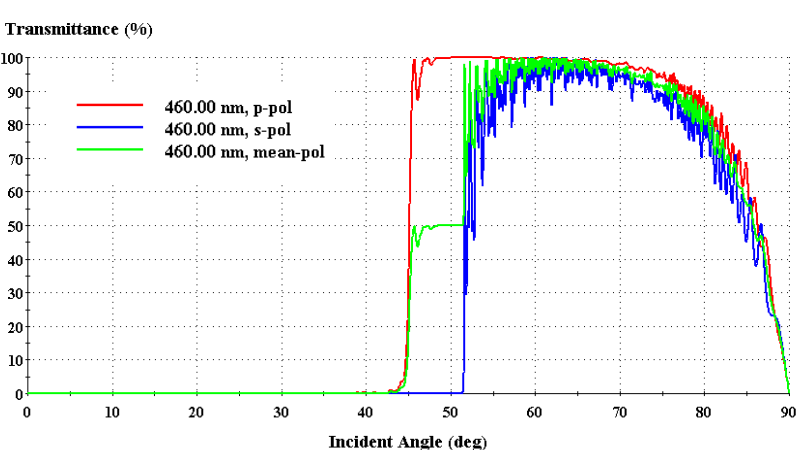

Figure 11: Transmittance versus incidence angle of the isotropic polymeric LED-masking filter at the central wavelength of $460 \mathrm{~nm}$

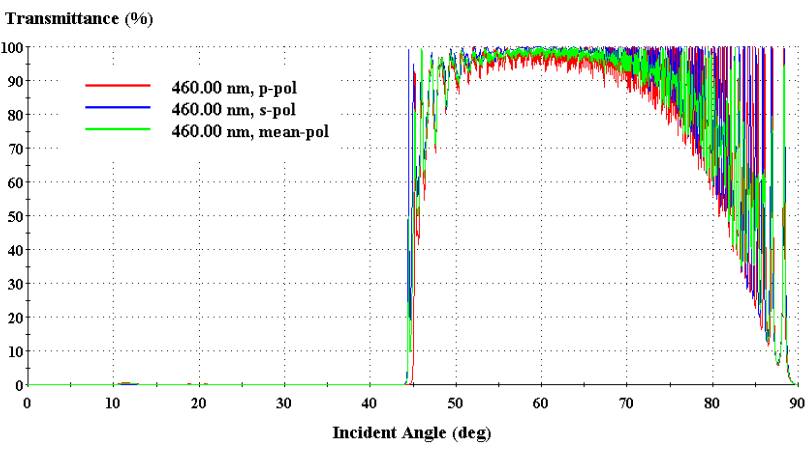

Figure 12: Transmittance of the birefringent polymeric LED-masking filter at the central wavelength of 460nm 


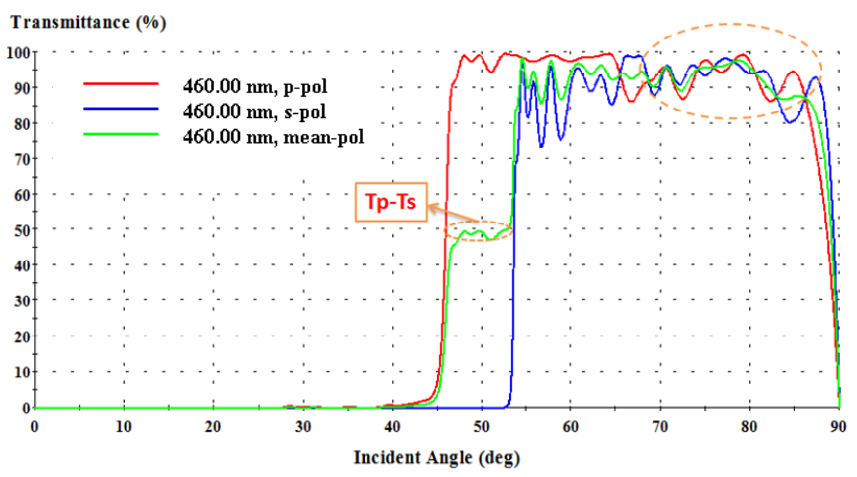

Figure 13 Transmittance versus incidence angle of the HML LED-masking filter at the central wavelength of 460nm

transmittance presented at $460 \mathrm{~nm}$ gives a smaller angular band edge difference of about $7^{\circ}$, and moreover effectively eliminates the oscillations at larger incident angles of the previous design. This demonstrates the great potential of the three-material multilayer to create a satisfying angular filter design. However, optical design and manufacturing are challenging.

\subsection{Simulation results for the Compact Collimator}

Optical ray tracing simulations were done with the improved angular filter designs. The calculated far field angular light distributions with $\mathrm{HL}$ filter $\left(\mathrm{Nb}_{2} \mathrm{O}_{5}\right.$ and $\left.\mathrm{SiO}_{2}\right)$, HML (three-material multilayer) and polymeric multilayer filter LEDmasking filter are shown in green, red and blue respectively in Figure 14(b). The output intensity along the normal has indeed been increased. The efficiency has been improved from $58 \%$ for the original HL filter to $66 \%$ for the HML filter and to $70 \%$ for the polymeric LED-masking filter. This verified that the new designed filters succeeded to increase the total output energy by having polarization equality. It is noted that it is not possible to perform ray tracing simulations with birefringent multilayers.

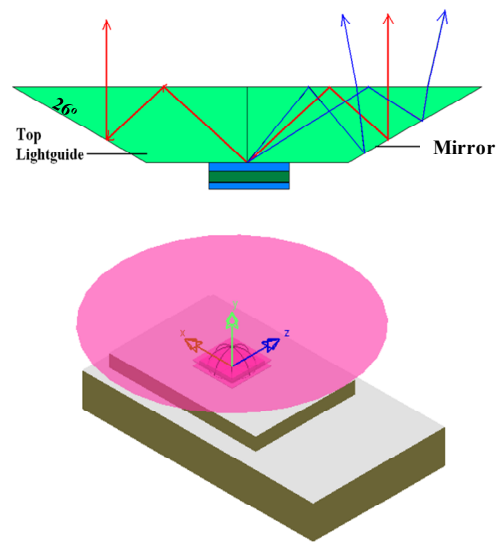

(a)

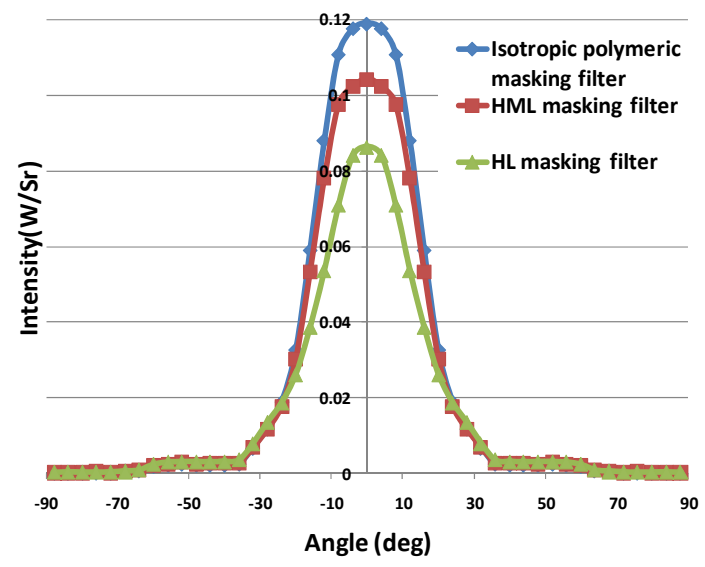

(b)

Figure 14: (a) The working principle of the compact collimator and the model in LightTools; (b) Calculated far field angular distribution of the compact collimator with three different filters.

\section{LARGE AREA PLANAR COLLIMATOR}

A simple multi-LED large-area planar collimator was designed by using another novel multilayer angular filter as shown in Figure 15. The LED sources are immersed at the bottom of a silicone rubber layer. The Lambertian distribution of the bare LED is changed into a collimated beam output after the filter. This overcomes the complicated lightguide shape and the precise alignment when combined with the LEDs. Light of small incident angles is transmitted by the filter, while light at large angles is reflected back and recycled by the $98 \%$ highly reflective white surface at the silicone bottom. The filter lies between the glass and the air. Light emitted from the LEDs at angles larger than the critical angle consequently 


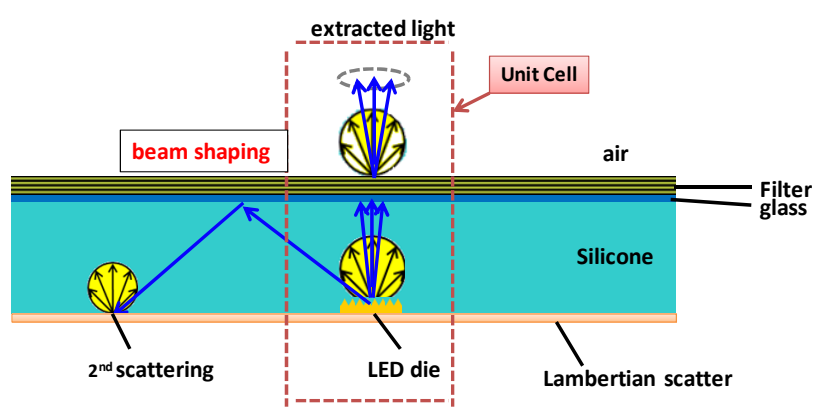

Figure 15: The new optical configuration of a large area planar collimator

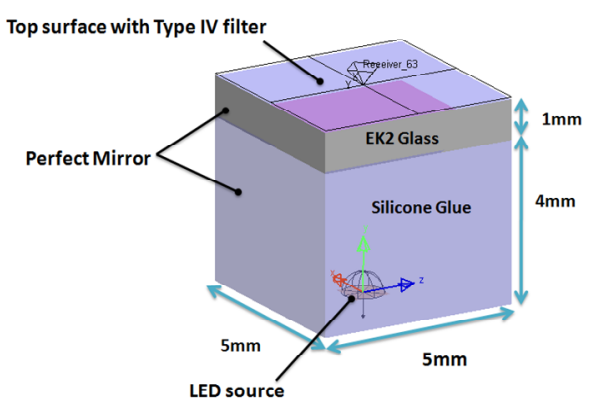

Figure 16: Unit cell model of the planar collimator in LightTools

suffers TIR and is reflected back. The ideal filter performance and target settings are $\mathrm{T}_{\mathrm{p}}=\mathrm{T}_{\mathrm{s}}=100 \%$, for $\theta=\left[0^{\circ}: 1^{\circ}: 8^{\circ}\right]$ and $\mathrm{T}_{\mathrm{p}}=\mathrm{T}_{\mathrm{s}}=0 \%$, for $\theta=\left[10^{\circ}: 1^{\circ}: 42^{\circ}\right]$. Nichia NS2B095AT blue medium power LEDs (central wavelength 468nm, FWHM of $25 \mathrm{~nm}$ ) are the selected light sources.

\subsection{Collimator filter design}

The collimator filter was designed with the available materials $\mathrm{SiO}_{2}\left(\mathrm{~L}, \mathrm{n}_{\mathrm{L}}=1.46\right)$ and $\mathrm{Ta}_{2} \mathrm{O}_{5}\left(\mathrm{H}, \mathrm{n}_{\mathrm{H}}=2.23\right)$ and restricted to a thickness of less than $3 \mu \mathrm{m}$. The initial filter design and performance optimization were achieved in Essential Macleod with the above target settings. The final filter is a 34-layer, 2.69um-thick HL multilayer film, with the p- and spolarized angular transmittance at the peak wavelength of $468 \mathrm{~nm}$ shown in Figure 17. Transmitted light smaller than $10^{\circ}$ is maximized and good reflection is obtained from $18^{\circ}$ to the critical angle. Highly directional light output cannot be achieved over the whole LED spectrum. Some of the long wavelength part of the LED spectrum is reflected instead of transmitted at small angles and hence will not emerge from the collimator. Instead, it leaks out at large output angles of around $80^{\circ}$, indicated in Figure 18 where for a wavelength of $481 \mathrm{~nm}$ a transmission peak is observed at angle close to the critical angle.

\subsection{Fabrication and Measurement}

The 34-layer collimator filter was deposited on a $0.7 \mathrm{~mm}$-thick EK2 glass of $50 \times 50 \mathrm{~mm}^{2}$ size by the thin film manufacturing facilities of Philips Innovation Services. The filter transmittance spectrum for various incident angles was measured by using a modified Perkin Elmer Spectrophotometer Lambda800 ${ }^{[8]}$ Comparison between the measured results in experiment and the calculated result from Essential Macleod were performed for incidence angles between $0^{\circ}$ to $40^{\circ}$ in glass and plotted in Figure 19. The measured result is in good agreement with the averaged calculated one, except for minor inequality from $0^{\circ}$ to $10^{\circ}$, which can be explained by the angular divergence of the measurement beam. From $0^{\circ}$ to $20^{\circ}$, within the FWHM of the LED spectrum, the light is highly transmitted, while from $20^{\circ}$ to $40^{\circ}$, LED light experiences a low mean transmittance.

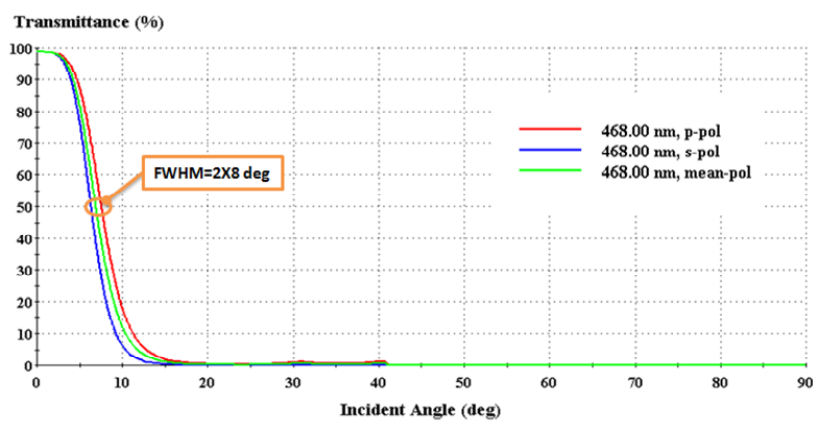

Figure 17: Transmittance versus incidence angle of the collimator filter at the central wavelength of $468 \mathrm{~nm}$ showing a FWHM of $16^{\circ}$

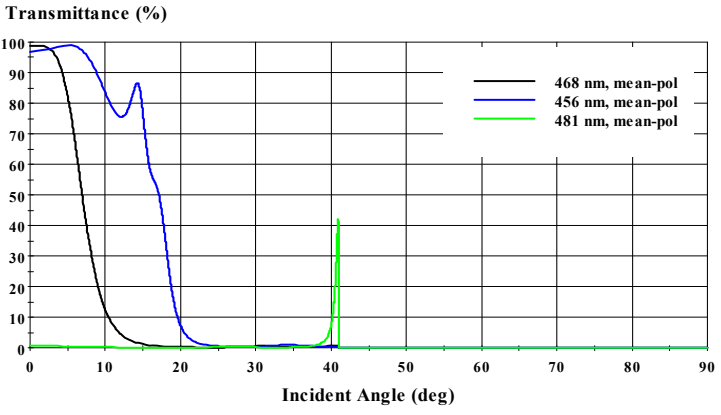

Figure 18: Transmittance versus incidence angle at three main wavelengths (central, $\pm \mathrm{FWHM} / 2$ ) of the Blue LED spectrum 

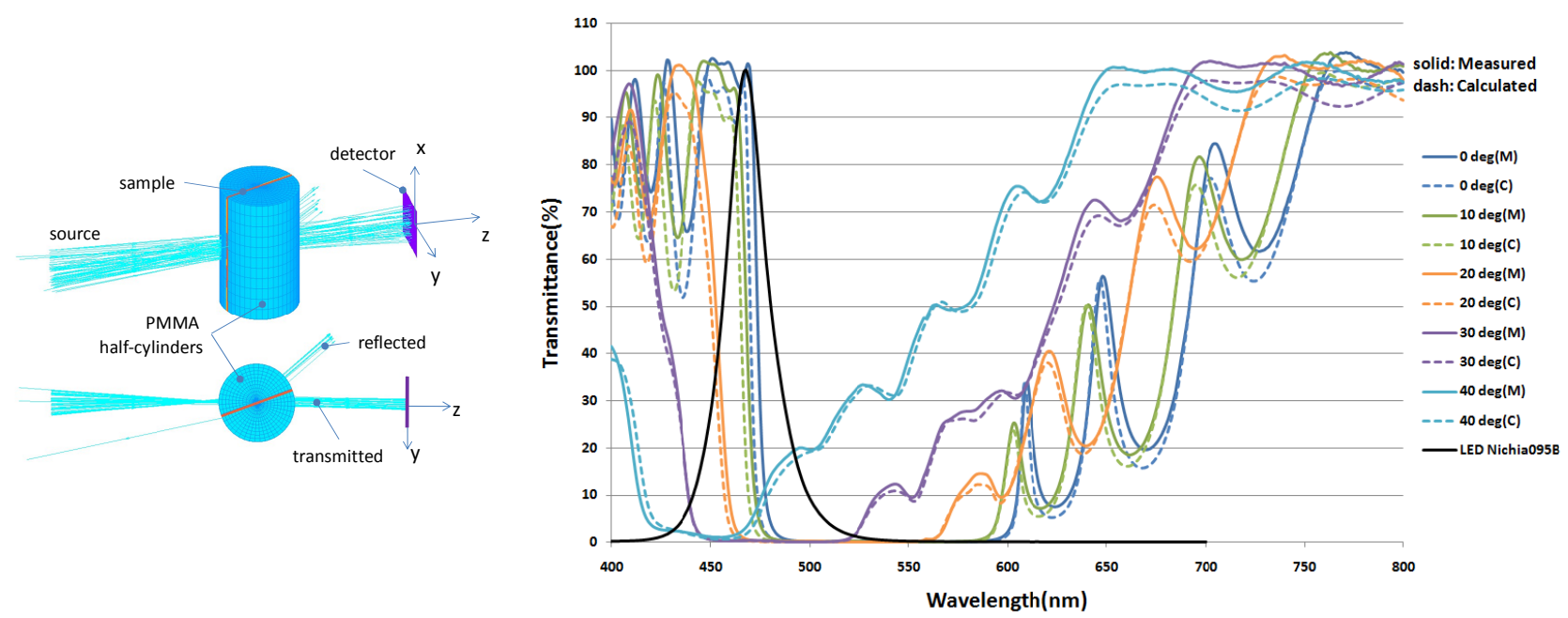

Figure 19: Left: schematic drawing of the modified spectro-photometer ${ }^{[8]}$ Right: Measured and calculated mean transmittance spectra at small incident angles. Angles are in glass. Solid lines refer to measured value and dashed lines refer to the calculated value. The black curve is the blue LED spectrum.

\subsection{Large Area Planar Collimator Sample}

The innovative large area planar collimating luminaire of Figure 15 was modeled using LightTools. The design was simplified into a single unit cell, by positioning only one LED and setting the boundary surfaces to be perfect mirrors, as illustrated in Figure 16. The bottom of the silicone glue is fully covered with white scattering paint of $98 \%$ reflectivity. The optical performance overview of the model without and with collimator filter is shown in Figure 20. The luminous intensity distribution without filter displays a good Lambertian pattern. The one with the collimator filter showed a collimated beam with a FWHM of $36^{\circ}$. According to the simulations, the luminous intensity along the normal increases more than threefold for these choices of dimensions and reflectivity, but the efficiency will be strongly dependent on reflectivity as shown in Figure 20(c). The true colour intensity distribution in Figure 20 (a) and (b) proves the beam collimating effect of the designed model.

Based on the optical performance prediction from ray tracing simulation, a testing prototype of the large area planar collimator was fabricated, as displayed in Figure 21(a). The filter glass lies on top and optically in contact with the 4-mm thick, 50x50 $\mathrm{mm}^{2}$ square dimension silicone rubber with nine Nichia NS2B095AT blue medium power LEDs irregularly located inside. The LED light is emitted from the top surface, while the other fives surfaces are covered with highly

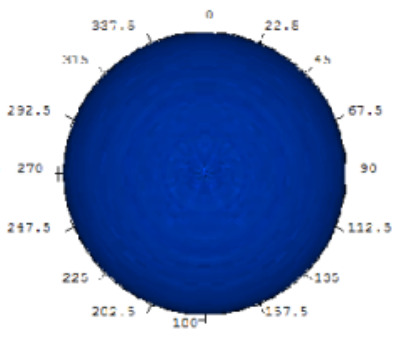

(a) without filter

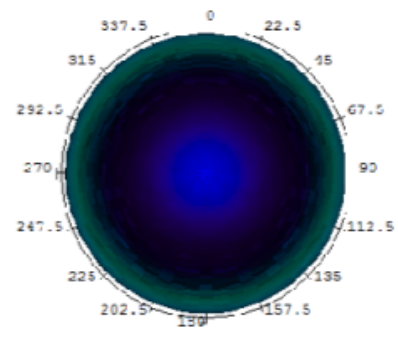

(b) with filter

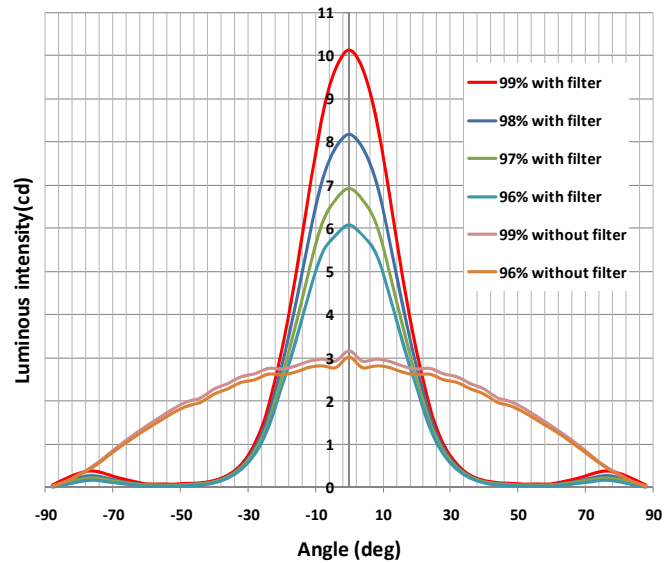

(c) cross sections for various reflectivities

Figure 20: Simulated true color luminous intensity angular distribution of the planar collimator 


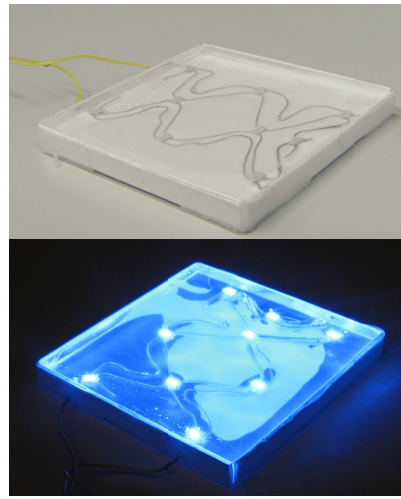

(a) without filter

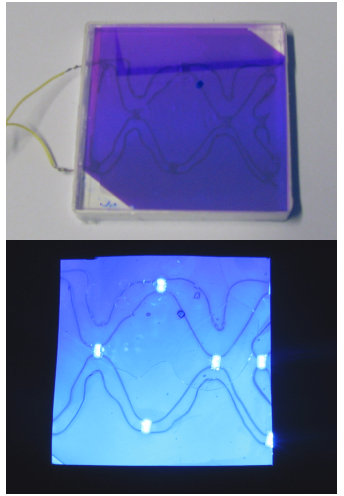

(b) with filter

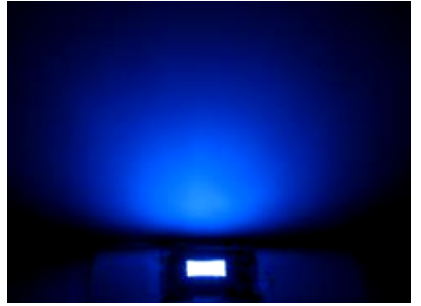

(c) without filter

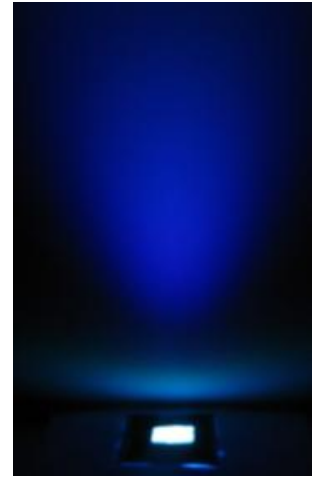

(d) with filter

Figure 21: (a,b) Pictures of the planar collimator prototype;

$(\mathrm{c}, \mathrm{d})$ the radiation luminous projection pattern.

reflective white polymeric sheet material to achieve light recycling. At first, the light distribution patterns were directly observed on a white diffusive screen perpendicular to the sample plane, without and with collimator filter respectively given in Figure 21(c,d). Clearly it can be seen that without the filter, light gives out a uniform blue Lambertian emission. The radiation has a collimated beam profile when the filter is applied. Greenish light leakage with low brightness was measured at large output angle from $70^{\circ}$ to $90^{\circ}$, which was also predicted with the LightTools simulation of Figure 20.

The luminance distribution for angles from $-80^{\circ}$ to $+80^{\circ}$ of a $4 \mathrm{~mm}^{2}$ area in-between the LEDs was measured with a conoscope EZContrast160R from ELDIM, shown in Figure 22 both without filter and with filter mounted on. It can be seen that light with FWHM of $36^{\circ}$ is obtained, including a greenish ring at larger angle. This is in good agreement with the simulation results of Figure 20(c).

Further comparison was done by measuring the spectra and efficiency of the planar collimator in an integrating sphere set-up. Figure 23 indicates the normalized radiant spectra with and without filter attached. It can be seen that for this LED type the spectrum shifts $4 \mathrm{~nm}$ to shorter wavelengths when the driving current increases from $5 \mathrm{~mA}$ to $60 \mathrm{~mA}$. The addition of the filter shifts the peak of the spectrum even further because it blocks the longer wavelengths. The efficiency, defined as the ratio of the measured flux with and without the filter is increasing with driving current as shown in Figure 24. It has a dependency on peak wavelength, from $21.8 \%$ to $27.7 \%$ ( $471.5 \mathrm{~nm}$ to $468 \mathrm{~nm}$ ). The efficiency and the normal luminous intensity can be enhanced by using white reflectors with higher reflectivity, seen from the calculated intensity performance in Figure 20(c). Some light absorption of the $\mathrm{Ta}_{2} \mathrm{O}_{5}$ material in the collimator filter also reduces the total efficiency. The LightTools calculated efficiency using a reflectivity of $98 \%$ is also shown in Figure 24. It behaves similarly to the measured results but it is lower than the measured value by a factor of 1.4 which may suggest a reflectivity of only $95 \%$ (see Figure 20 ) or some absorption in the silicone.

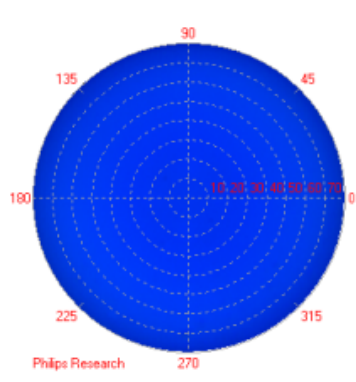

(a) without filter

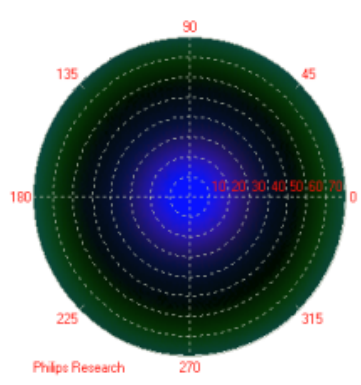

(b) with filter

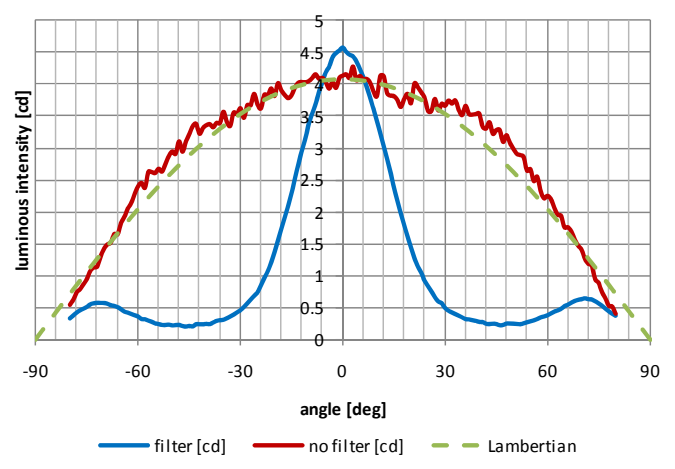

(c) cross sections of the measured luminous intensity

Figure 22: Measured angular characteristics of the luminance and intensity distribution of a small part of the planar collimator prototype 


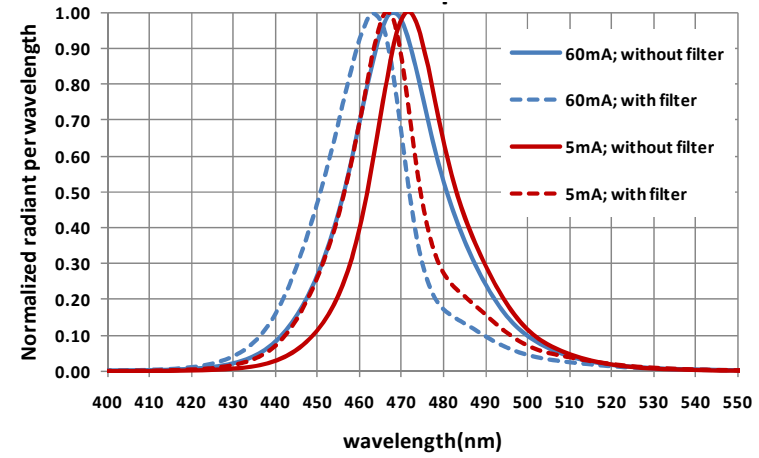

Figure 23 Measured normalized radiant spectra of the planar collimator. Blue for $60 \mathrm{~mA}$, red for $5 \mathrm{~mA}$; solid without filter, dashed with filter

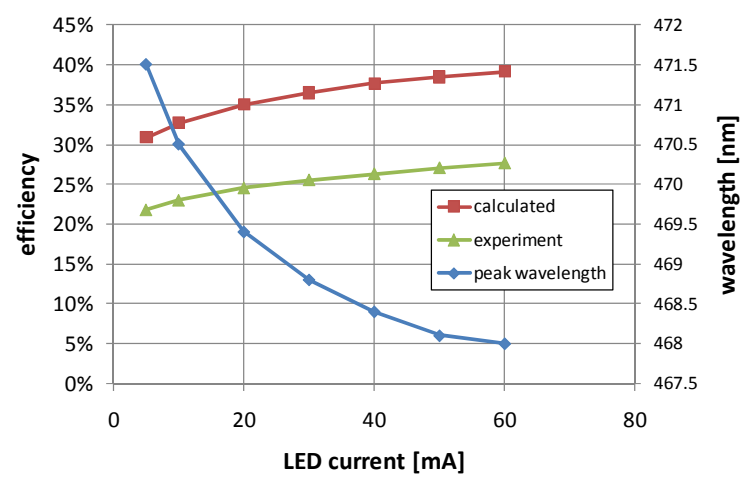

Figure 24 Measured and calculated efficiency at different driving current per LED. In blue the measured peak wavelength is plotted.

\section{CONCLUSION}

The use of interference filters as angular recycling filters offers many interesting possibilities for compact collimators. A first type of filter that masks the LED and transmits light at angles larger than the critical angle has been improved by using polymeric multilayer stacks or by using more than just 2 materials in the interference stack. The concept is not limited to blue LEDs. It has already been proven that interference angular filters for red and green LEDs can be designed as well. ${ }^{[2]}$

A second type of filter complementarily transmits light for small angles of incidence, thus enabling a novel large area planar collimated light source. We see good agreement between our simulation results and measurements on prototypes. The planar collimator system is scalable, simply by increasing the number of embedded LEDs and/or the size of the light guide. Beneficially, the LED positions are not fixed and the distance between one another is inconstant and flexible. The planar collimator system can be simply manufactured without any precise alignment and complicated shaped reflecting coating.

\section{Acknowledgments}

We acknowledge the help of Kai Yuan Chi in optical simulations. Serge Bierhuizen, Eugen Onac, Henri Jagt, Cor de Haas, Thomas Diederich, Gilbert Verbeek and Keesjan Niemantverdriet are acknowledged for realizing the prototypes. We thank Florian Bociort and Paul Urbach from the Delft University for helpful discussions.

\section{REFERENCES}

[1] Serge Bierhuizen, Michael Krames, Gerard Harbers, Gon Weijers, "Performance and trends of high power Light Emitting Diodes", SPIE 6669, 66690B (2007).

[2] C.H. Ho, H.J. Cornelissen, M.P.C.M. Krijn, and H.A. van Sprang, "Multilayer-Incoupling-Structure Design for Ultra-Thin LED-Backlights", International Display Workshop 2008 IDW08, 757-760 (2008).

[3] H.A. Macleod, [Thin-Film Optical Filters], 3rd ed., Inst. of Phys., London, (2001).

[4] Julio C. Chaves, Waqidi Falicoff, Bill Parkyn, Pablo Benítez, Juan C. Miñano, "Increased brightness by light recirculation through an LED source", Proc. of SPIE 7059, 705902-1(2008)

[5] Timothy J. Nevitt, Timothy J. Hebrink, Michael F. Weber, "Thin hollow backlights with beneficial design characteristics", US 2010/0136953 A1, (2010).

[6] John A. Wheatley, Gilles J. Benoit, James E. Anderson, Rolf W. Biernath, David G. Freier, Thomas R. Hoffend, C. David Hoyle, Tao T. Liu, Jenna D. Lu, Michael A. Meis, Vadim V. Savvateev, Craig R. Schardt, Matthew E. Sousa, Michael F. Weber, and Timothy J. Nevitt, "Efficient LED light distribution cavities using low loss, angle-selective interference transflectors", Optics Express 17, Issue 13, 10612-10622 (2009).

[7] Michael F. Weber, Carl A. Stover, "Giant Birefringent Optics in Multilayer Polymer Mirrors", Science 287, 2451$2456(2000)$.

[8] Hugo J. Cornelissen, Chenhung Ho, Haiyan Ma, Marcel P.C.M. Krijn, Hans A. van Sprang "Injecting light of high power LEDs into Thin Light Guides", Proc. of SPIE 7652, 765212 (2010). 